\title{
Ocultamiento del Mensaje de Señales de Voz usando la Transformada de Ondita Haar Discreta
}

\author{
Alejandro Martínez ${ }^{1}$, Zaira Pineda ${ }^{1}$ y Alberto Ramos ${ }^{2}$ \\ (1) Universidad Autónoma de San Luis Potosí, Coordinación Académica Región Altiplano, Carretera \\ a Cedral km 5+600 Ejido San José de Las Trojes, 78700 Matehuala, San Luis Potosí-México \\ (e-mail: alejandro.martinez@uaslp.mx) \\ (2) Benemérita Universidad Autónoma de Puebla, Escuela de Ciencias de la Electrónica, Av. San \\ Claudio S.N., Ciudad Universitaria Puebla, Puebla-México
}

Recibido Abr. 21, 2009; Aceptado Jun. 15, 2009; Versión Final recibida Jul. 31, 2009

\begin{abstract}
Resumen
Este trabajo presenta un método de ocultamiento del mensaje para transmisión de señales de voz utilizando codificación por medio de bancos de filtros multi-resolución de análisis y de síntesis para la Transformada de Ondita Haar Discreta. Por medio de un banco de filtros Haar multi-resolución se realiza la separación de la señal en sus componentes y después se realiza una distorsión reversible de la síntesis multi-resolución de sus señales componentes, obteniendo una señal de salida que, aunque mantiene la misma información de la señal de entrada, el mensaje de la señal de voz que esta porta queda oculto en ella misma. Este método puede aplicarse para la transmisión codificada de señales de voz ya sea en su forma digital o analógica, y por su simplicidad resulta adecuado para implementarse en sistemas electrónicos digitales, ya sea como un arreglo de compuertas programables por campo, o como circuito integrado de aplicación específica.
\end{abstract}

Palabras clave: ocultamiento de mensaje, Transformada de Ondita Haar Discreta, codificación de señales de voz

\section{Message Concealment of Voice Signals using the Discrete Haar Wavelet Transform}

\begin{abstract}
This work introduces a message concealment method for voice signals transmission using codification through multi-resolution filter banks implementation for analysis and synthesis for the Discrete Haar Wavelet Transform. Through these multi-resolution filter banks of Haar wavelet the voice signal can be split in its components, and later a reversible distortion of the synthesis of its multi-resolution component signals is made, obtaining an output signal that, although maintains the same information of the original input signal, hides the message of the carried voice signal in itself. This method can be applied in codified transmission of voice, analog or digital signals, and due to the simplicity of the method it's suitable to be implemented on digital electronic systems like FPGA, or ASIC.
\end{abstract}

Keywords: message concealment, Discrete Haar Wavelet Transform, voice signals encoding 


\section{INTRODUCCIÓN}

La transformada de ondita Haar discreta ha sido ampliamente utilizada en la codificación y ocultamiento de datos de señales digitales (Agaian et al., 2006; Agbinya, 1996; Porwik y Lisowska 2004). Una forma de realizar la Transformada de Ondita Haar Discreta sobre una señal digital $x(i)$, $i=1,2, \ldots, n$, es separándola en dos señales con la mitad de longitud, llamadas señal de tendencia $x_{a}(j)$ y señal de fluctuación $x_{d}(j)$, siendo $j=1,2, \ldots, n / 2$, como se hace en Welstead (1999). La señal de tendencia es obtenida mediante el promedio de dos muestras consecutivas, tomadas de dos en dos, y la señal de fluctuación es tomada como la diferencia media de dos muestras consecutivas tomadas de dos en dos. Si la señal de tendencia es descompuesta también en dos señales, una de tendencia y la otra de fluctuación, tendremos como resultado la descomposición de la señal original $x(i)$ en tres señales: una señal de fluctuación $x_{d 1}(j)$, una señal de tendencia $x_{a 2}(k)$ y una señal de fluctuación $x_{d 2}(k)$, donde $k=1,2, \ldots n / 4$. Cuando una señal es descompuesta en esta forma se dice que se ha realizado sobre ella un análisis Haar multi-resolución de dos niveles. El análisis multi-resolución puede extenderse a más niveles. Si se requiere reconstruir la señal a partir de sus dos señales componentes, se reconstruyen dos muestras consecutivas $x(i-1)$ y $x(i)$ de acuerdo a las ecuaciones (1) y (2), con $i=2 j, y j=1,2, \ldots, n / 2$.

$$
\begin{aligned}
& x(i-1)=x_{a}(j)-x_{d}(j) . \\
& x(i)=x_{a}(j)+x_{d}(j) .
\end{aligned}
$$

Algunos sistemas digitales de codificación de señales emplean un banco de filtros multi-resolución de análisis con la transformada de onditas para descomponer una señal y los coeficientes de estas señales componentes son reordenados para su almacenamiento o para su transmisión (Sadkhan et al., 2007; Elfouly et al., 2008). Para decodificar a la señal se emplea primero el reordenamiento de los coeficientes y las señales componentes entran a un banco de filtros multi-resolución de síntesis con la transformada de ondita.

El objetivo del trabajo que se presenta ha sido desarrollar un método de codificación para ocultar el mensaje de las señales de voz diferente a los métodos ya existentes, con la posibilidad de ser implementado como sistema digital en tiempo real. Para lograr este objetivo se propone el empleo de la Transformada de Onditas Haar Discreta, aplicándola de forma tal que el mensaje de la señal de voz es ocultado en un ruido aparente proveniente de una las señales producto de la descomposición multi-resolución con la Transformada de Onditas Haar. Nuestro método es diferente de los métodos para el ocultamiento de datos encontrados en la literatura científica, como los de marca de agua y los esteganográficos (Cai y Zhao, 2004; Delforouzi y Pooyan, 2008), los cuales ocultan un mensaje codificándolo sobre una señal dada, de manera que el mensaje oculto que ella porta sea imperceptible. En las secciones siguientes se describe el método propuesto, se presentan y discuten los resultados obtenidos y se dan las conclusiones de este trabajo.

\section{MÉTODO DE OCULTAMIENTO DEL MENSAJE EN LA SEÑAL}

Supóngase que se cuenta con una señal analógica continua $x(t)$, con $t_{1} \leq t \leq t_{2}$, la cual se somete a un proceso de discretización y cuantización para obtener una señal digital $x(i)$, con $i=1,2, \ldots, n$, esto es, se realiza en $x(t)$ una conversión de un formato analógico a un formato digital, a una razón de muestreo de $F_{s}=n /\left(t_{2}-t_{1}\right)$ muestras cada segundo. La señal $x(i)$ se toma como señal de entrada a un banco multi-resolución de análisis con la ondita discreta Haar de cuatro niveles para obtener las señales componentes $x_{d 1}(j), x_{d 2}(k), x_{d 3}(l), x_{d 4}(m)$ y $x_{a 4}(m)$, donde $j=1, \ldots, n / 2 ; k=1,2, \ldots, n / 4$; $l=1,2, \ldots, n / 8 ;$ y $m=1,2, \ldots, n / 16$. A medida que la señal se separa en sus señales componentes, la información de voz que poseen esas señales componentes va disminuyendo. Si se reproduce cada una de esas señales componentes interpolándolas para que cada una de ellas tenga una razón de muestreo $F_{s}$ y se convierten en señales analógicas, se reconocerá en ellas parte de la información de la señal de voz $x(t)$, en particular, se podrá reconocer el mensaje de voz en las señales $x_{d 1}(j), x_{d 2}(k)$, $x_{d 3}(l), x_{d 4}(m)$ y $x_{a 1}(j), x_{a 2}(k), x_{a 3}(l)$. En resultados experimentales de este trabajo se encuentra que la interpolación de la señal de aproximación $x_{a 4}(m)$ obtenida en el cuarto nivel de análisis, produce una señal $x_{a 4}(t)$ en la cual ya no se reconoce el mensaje de voz, de manera que si se transmitiera sola esta señal componente $x_{a 4}(m)$ en formato digital, ó $x_{a 4}(t)$ en forma analógica, el mensaje de voz no 
podría ser recuperado. Si se sintetiza la señal original a partir de sus señales componentes Haar multi-resolución de cuatro niveles se recuperará una versión retardada $x(i-h)$ de la señal original $x(i)$, donde $h=16$, y convirtiéndola a la forma analógica se obtendrá una señal $x\left(t-t_{d}\right)$.

El método de codificación que se propone realiza una distorsión reversible en la señal original digital $x(i)$. Este método no se basa en la transmisión de los componentes Haar de la señal sino en una versión de la síntesis de estos componentes, distorsionada en una forma reversible que se obtiene al multiplicar cada una de las señales componentes multi-resolución Haar por una constante, obteniendo versiones escaladas de cada una de las señales componentes como se muestra en el conjunto de ecuaciones (3).

$x_{d i}^{\prime}(i)=D_{i} x_{d i}(i), \quad x_{d 2}^{\prime}(j)=D_{2} x_{d 2}(j), \quad x_{d 3}^{\prime}(k)=D_{3} x_{d 3}(k), \quad x_{d 4}^{\prime}(I)=D_{4} x_{d 4}(I), \quad x_{a 4}^{\prime}(I)=A_{4} x_{a 4}(I)$.

Estas señales componentes escaladas se utilizan después como señales de entrada a un banco de filtros multi-resolución de síntesis con la transformada de ondita Haar, formando así una versión distorsionada $x^{\prime}(i-h)$ de la señal de entrada $x(i)$, como se muestra en la Fig.1.

Si $A_{4}=1$ y $D_{i}=1$, para $i=1, \ldots, 4$, entonces, a la salida del banco de filtros multi-resolución de síntesis se tendrá una señal de salida $x(i-h)$, que es una versión reconstruida de $x(i)$, retardada en $h$ muestras. Pero si esta condición no se cumple, se tendrá a la salida una señal $x$ '(i-h) que es una versión retardada y distorsionada de la señal de entrada. Diversas combinaciones de estos coeficientes producen a la salida del codificador versiones distorsionadas de la señal de entrada, y en algunas de ellas se reconoce el mensaje de la señal de voz.

Para lograr obtener una señal $x^{\prime}(i-h)$ en la que pueda ocultarse el mensaje de la voz es necesario seleccionar adecuadamente los coeficientes $D_{1}, D_{2}, D_{3}, D_{4}$ y $A_{4}$. La propuesta de este trabajo consiste en realizar el ocultamiento del mensaje de voz, que mayormente portan las señales $x_{d 1}, x_{d 2}$, $x_{d 3}$, y $x_{d 4}$, dentro de la señal componente $x_{a 4}$ porque esta señal componente ya no porta mensaje de voz, de manera que al realizar la amplificación de esta señal con respecto a las demás componentes se logrará ocultar el mensaje de la señal de voz dentro de un ruido aparente producido por $x_{a 4}$. Para reconstruir una versión retardada $x(i-p)$ de la señal de $\operatorname{voz} x(i)$, donde $p=2 h$, en la cual se puedan recuperar las características acústicas de la señal original de entrada y el mensaje que ella porta, se usa un banco de filtros de ondita Haar multi-resolución para hacer análisis-distorsión-síntesis en una forma similar a como se hace en la etapa de codificación, pero en este banco los coeficientes de escalamiento $D_{1 d e c}, D_{2 d e c}, D_{3 d e c}, D_{4 d e c}$ y $A_{4 d e c}$, de las señales de la descomposición con la transformada de onditas, son los inversos multiplicativos de aquellos que se utilizan en la etapa de codificación, como se indica en las ecuaciones (4).

$D_{1 d e c}=1 / D_{1}, \quad D_{2 d e c}=1 / D_{2}, \quad D_{3 d e c}=1 / D_{3}, \quad D_{4 d e c}=1 / D_{4}, \quad A_{4 d e c}=1 / A_{4}$.

El proceso de decodificación descompone a la señal distorsionada en sus señales componentes, revierte la distorsión producida en la codificación quitando el escalamiento en ellas y las reintegra por medio de un banco de filtros multi-resolución de síntesis para la transformada de ondita Haar. A la salida del decodificador se obtiene una señal que es una versión retardada de la señal original que entró en el codificador, logrando la reconstrucción perfecta de la señal y su mensaje. La Fig. 2 muestra a bloques los métodos de codificación y de decodificación propuestos. Entre el codificador y el decodificador aparece un bloque denominado retardo de sincronización, que introduce un retardo de $B$ muestras en la señal de salida del codificador. Este bloque sirve para modelar el retardo que puede intervenir en la señal desde su salida del codificador hasta su llegada al decodificador. Solamente si $B=C h$, con $C=1,2, \ldots$, la señal de salida será $x(i-2 h-B)$, obteniéndose una réplica retardada de la señal de entrada $x(i)$, en caso contrario la señal de entrada no es decodificada.

\section{RESULTADOS Y DISCUSIÓN}

La determinación de los valores de los coeficientes de escalamiento que logran producir una señal de salida en la que se oculte el mensaje de voz se realizó de manera experimental, y los resultados obtenidos se muestran en la tabla 1 . Mientras más grande sea el coeficiente $A_{4}$ en relación con el valor máximo de los coeficientes $D_{i}$, con $i=1,2,3,4$, el mensaje de voz de la señal $x^{\prime}(i-h)$ se oculta 
en mejor forma. De acuerdo con los datos de la tabla 1, el mensaje de la señal de voz x'(i-h) no se reconocerá si se cumplen las relaciones (5).

$A_{4} \geq 512 D_{i}$, para $i=1,2,3,4$.

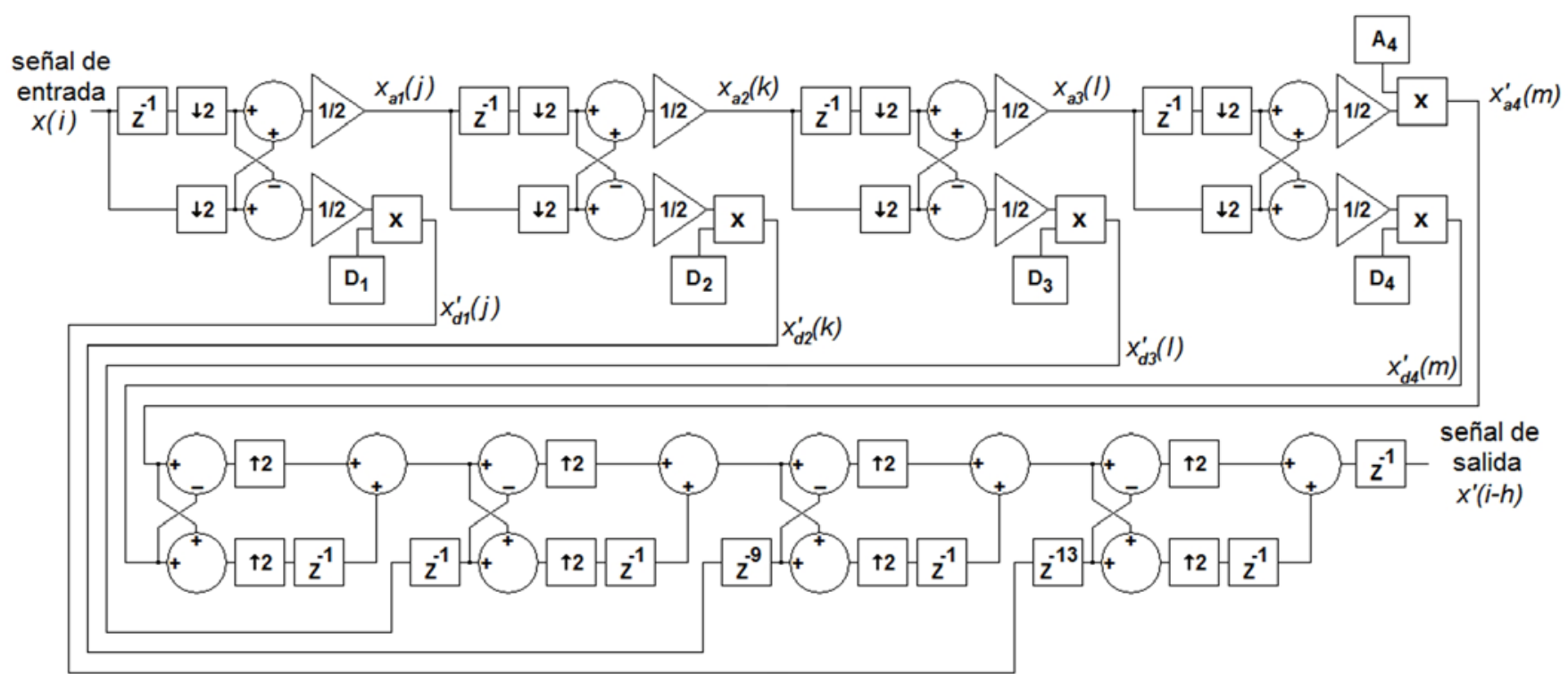

Fig. 1: Diagrama a bloques que ilustra el método de ocultamiento del mensaje de señales de voz usando la transformada de ondita Haar discreta multi-resolución de cuatro niveles.

El codificador de ocultamiento de mensaje de la Fig. 1 y el decodificador correspondiente fueron implementados y probados usando la herramienta SIMULINK. Las señales de entrada utilizadas fueron señales de voz en idiomas español e inglés, grabadas en archivos en formato .wav con una duración de 15 segundos, con frecuencia de muestreo $F_{s}=8000$ muestras/segundo y muestras codificadas con 8 bits en cada señal. En SIMULINK se realizó la conversión de la señal contenida en el archivo de audio a un vector $x$ (i) con $i=1,2, \ldots, 120000$, que sirve como señal de entrada al sistema codificador de ocultamiento. La señal fue procesada utilizando diferentes valores para los coeficientes $A_{4}$ y $D_{i}$, para $i=1, \ldots, 4$. La señal de salida $x^{\prime}(i-h)$ obtenida a la salida del codificador fue escalada para acondicionarla al formato .wav y fue grabada y ejecutada con SIMULINK y también con el reproductor de audio Windows Media. Se realizaron pruebas de percepción del mensaje con un grupo de 20 personas, a las que se pidió que identificaran el mensaje en la señal de salida. Se utilizaron diferentes mensajes de voz que incluían voces de niño, de mujer, y de hombre.

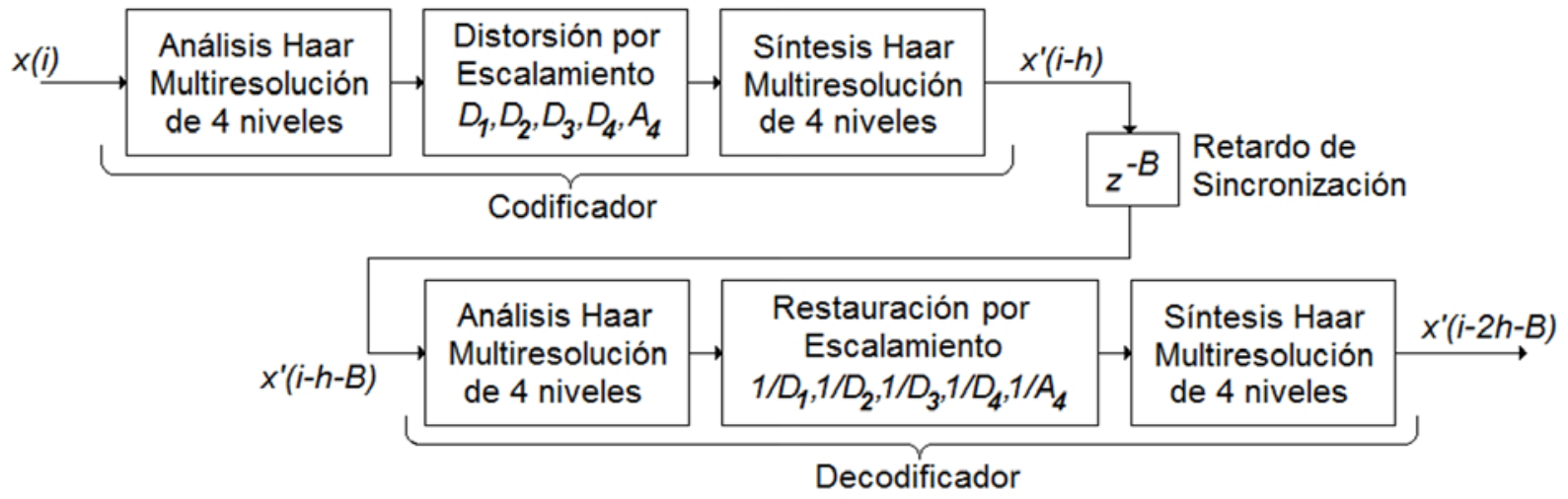

Fig.2: Diagrama a bloques de los métodos de codificación y de decodificación propuestos.

Al incrementarse sobre la unidad la razón $A_{4} / \max \left(D_{i}\right)$, la señal de salida del codificador pierde algunas características acústicas, hasta que al cumplirse las relaciones (5) no se percibe el mensaje de la señal de voz en la señal de salida distorsionada. El mensaje se encuentra oculto en un aparente ruido que resulta de la amplificación de la señal de tendencia $x_{a 4}(l)$ que ya tiene poca 
información de voz. Este método fue probado también utilizando bancos de análisis y de síntesis de una etapa, y bancos multi-resolución de análisis y de síntesis de dos, tres, y cuatro etapas con la transformada de ondita Haar. El método solamente funciona para bancos de filtros multi-resolución de cuatro etapas. En las implementaciones de una, dos y tres etapas no se logra ocultar el mensaje porque las señales de tendencia $x_{a 1}(j), x_{a 2}(k)$ y $x_{a 3}(l)$ todavía conservan suficiente información de voz, y no logran generar por sí mismas el ruido aparente en el que se oculta el mensaje de las otras señales de la descomposición multi-resolución.

Tabla 1: Percepción del mensaje en función de los valores relativos de $A_{4}$ y el máximo valor de $D_{i}$

\begin{tabular}{|c|c|c|c|c|}
\hline \multirow{2}{*}{$A_{4} / \max \left(D_{i}\right)$} & \multicolumn{4}{|c|}{ Percepción del mensaje en diferentes tipos de voz } \\
\cline { 2 - 5 }$i=1,2,3,4$ & Voz de niño & Voz de mujer & Voz aguda hombre & Voz grave hombre \\
\hline 0 & sí & sí & sí & sí \\
\hline 1 & sí & sí & sí & sí \\
\hline 2 & sí & sí & sí & sí \\
\hline 4 & sí & sí & sí & sí \\
\hline 8 & sí & sí & sí & sí \\
\hline 16 & sí & sí & sí & sí \\
\hline 32 & sí & sí & sí & sí \\
\hline 64 & débil & débil & sí & débil \\
\hline 128 & débil & débil & débil & no \\
\hline 256 & no & no & no & no \\
\hline 512 & no & no & no & no \\
\hline 1024 & no & no & no & no \\
\hline 2048 & no & no & no & no \\
\hline 5096 & no & no & & \\
\hline
\end{tabular}

Se probaron en el decodificador diferentes combinaciones de los coeficientes de escalamiento $D_{1 \text { dec, }}$ $D_{2 d e c}, D_{3 d e c}, D_{4 d e c}$ y $A_{4 d e c}$, y solamente su logra reconstruir la señal en forma perfecta cuando se cumplen las ecuaciones (4). Se probaron diferentes valores del retardo $B$ con que la señal procedente del codificador llega al decodificador, y solamente se realiza la decodificación si este retardo es un múltiplo entero positivo del retardo producido por el codificador, esto es $B=C h$, donde $h=16$ y $C=0,1,2, \ldots$, . A la entrada del decodificador deberá estar presente una señal de la forma $x^{\prime}(i-h-C h)$ para que el decodificador recupere una señal $x(i-2 h-C h)$ que sea una réplica retardada de la señal de entrada $x(i)$ en el codificador. El bloque de retardo $B$ de sincronización puede añadirse a la salida del codificador para introducir un retardo adicional no múltiplo del retardo $h$ que produce el codificador, de tal manera que si la señal de salida del codificador es grabada o transmitida, entonces solamente puede ser decodificada y reproducida si el decodificador primero compensa este retardo añadiendo retardos adicionales para hacer que el retardo total a su entrada sea un múltiplo entero positivo de $h$, haciendo más robusto el método. La Fig. 3 muestra la gráfica de la señal $x(i)$ de entrada al codificador y la señal $x(i-2 h-C h)$ reconstruida en el decodificador con $C=0$.

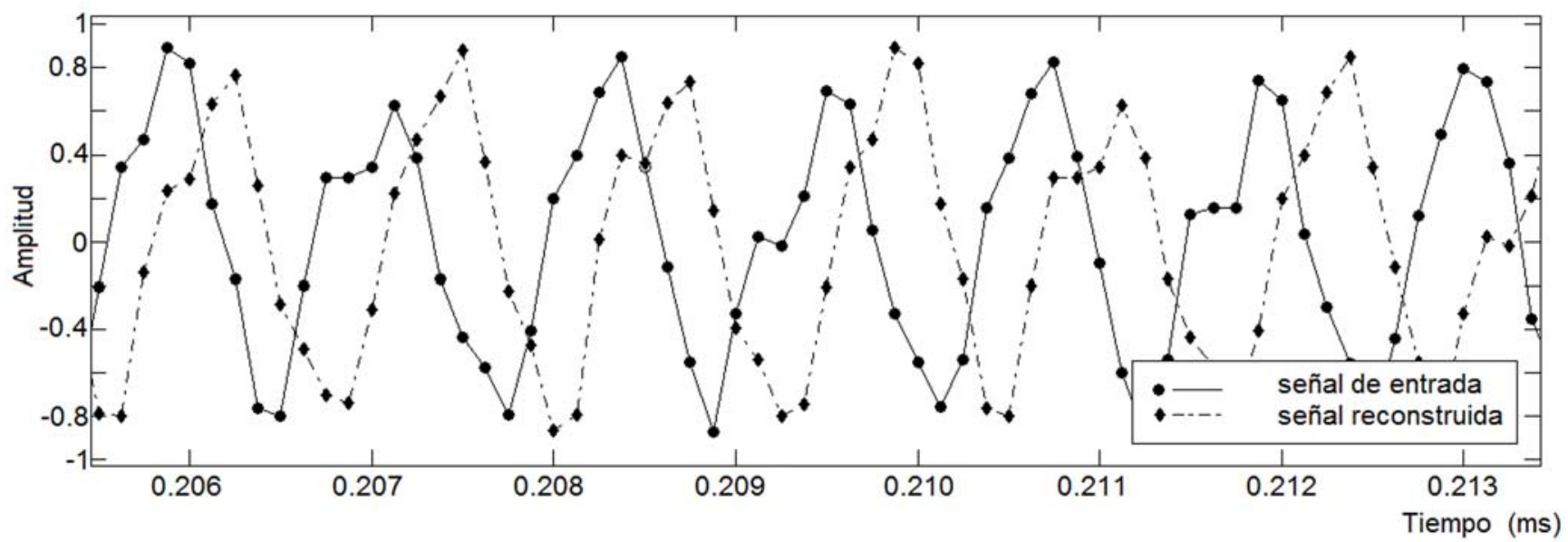

Fig.3: Señales de entrada al codificador y de salida del decodificador con amplitud de formato .wav 


\section{CONCLUSIONES}

En este trabajo se ha presentado un método de ocultamiento de señales de voz que se diferencia de los de marca de agua y los esteganográficos en que este realiza el ocultamiento del mensaje de la señal de voz dentro de la información perteneciente a la propia señal de entrada, produciendo una versión distorsionada de la señal original que se envuelve en un ruido aparente proveniente de sí misma. También se ha presentado el método de decodificación de la señal oculta, similar al método de codificación, y se han mostrado las condiciones en que se logra reconstruir a la señal de entrada y su mensaje en forma perfecta, describiendo también el efecto del retardo entre el codificador y el decodificador y que este retardo puede aprovecharse para hacer más robusto el método propuesto.

Por la sencillez de los bancos de filtros multi-resolución de análisis y de síntesis, y por el poco retardo producido en la señal de salida con respecto a la señal de entrada, este método es adecuado para implementarse como sistema electrónico digital con procesamiento en tiempo real, ya sea basado en arreglos de compuertas programables por campo o como circuito integrado de aplicación específica.

\section{AGRADECIMIENTOS}

Este trabajo es financiado por PROMEP-SEP a través del proyecto PROMEP/103.5/08/3123 y es financiado también por el FAI de la UASLP, a través del proyecto con convenio C08-FAI-10-30.66

\section{REFERENCIAS}

Agaian, Sos S., y otros cuatro autores; Golden Ratio-Haar Wavelet Based Steganography, Proceedings of the 2006 International TICSP Workshop on Spectral Methods and Multirate Signal Processing, SMMSP2006, Florence, Italy, September 2006. TICSP, pp. 121-126, (2006).

Agbinya, J.I.; Discrete wavelet transform techniques in speech processing, Proceedings. 1996 IEEE TENCON. Digital Signal Processing Applications: 2, 514-519 (1996).

Cai, Libin y Jiying Zhao; Evaluation of speech quality using digital watermarking, IEICE Electron. Express; 1(13), 380-385 (2004).

Delforouzi, Ahmad y Mohammad Pooyan; Adaptive Digital Audio Steganography Based on Integer Wavelet Transform, Circuits, Systems, and Signal Processing, Birkhäuser Boston: 27(2), 247-259 (2008).

Elfouly, Fatma H., Mohamed I. Mahmoud, Moawad I. M. Dessouky, y Salah Deyab; Comparison between Haar and Daubechies, Wavelet Transformations on FPGA Technology, International Journal of Computer, Information, and Systems Science, and Engineering: 2, 1 (2008).

Porwik, Piotr y Agnieszka Lisowska; The Haar-Wavelet Transform in Digital Image Processing: Its Status and Achievements, Machine GRAPHICS \& VISION: 13(1-2), 79-98 (2004).

Sadkhan, S.B., N. Abdulmuhsen y N.F. Al-Tahan; A Proposed Analog Speech Scrambler Based on Parallel Structure of Wavelet Transforms, National Radio Science Conference, 2007. NRSC 2007, pp. 1-12 (2007).

Welstead, Stephen; Fractal and Wavelet Image Compression, Tutorial Text in Optical Engineering, Volume TT40, SPIE Optical Engineering Press, pp 93-117 (1999). 